\title{
Determining the requirements related to the design and operation of rail vehicle simulators
}

\author{
M. Bajerlein, J. Merkisz \& Ł. Rymaniak \\ Institute of Combustion Engines and Transport, \\ Poznan University of Technology, Poland
}

\begin{abstract}
The paper presents the most currently used driving simulators. Based on the analysis of the operating conditions of the rail vehicles the criteria to be met by this type of vehicle simulator have been characterized. In addition, the paper defines the animation requirements. Reference was made to both graphics and video presentation techniques. The paper also discusses the design requirements for the installation of a cab simulator and extras such as the sound effects. The most important requirements have been defined for programming of the simulated routes, weather conditions, collisions and other parameters that can significantly improve the quality of a training simulator. The paper discusses the function of control of the course of the exercise trainer and the requirements of his job. Finally the paper discusses the issues of the negative impact of driving simulators on the human body and methods of prevention thereof.

Keywords: animation, simulation disease, driving simulator, rail vehicle simulator.
\end{abstract}

\section{Introduction}

A locomotive is a unique railroad vehicle designed to haul or push large tonnages (up to 4000 tons) carried on cargo or passenger railcars. Depending on their purpose locomotives are fitted with different powertrains of the power output from 1000 to $26000 \mathrm{~kW}$. The maximum speeds reached by locomotives are $500 \mathrm{~km} / \mathrm{h}$ plus. The mentioned features and operating conditions require special skills from the motorman. High vehicle inertia is one of the main factors that make the operation of the vehicle a complex issue that requires appropriate prediction skills. The simulation of the rail vehicle needs to be based not only on 
the said conditions but also certain requirements that are related to the locomotive design and the reflection of the actual operating conditions or simulation of certain driving situations.

\section{Requirements for the design of a rail vehicle simulator}

The design of simulators varies widely. We can distinguish them according to the types of systems directly generating the animation or the characteristics of the spatial vision. As for the locomotive simulator the most appropriate solution is the application of a computer system. The computer allows a mathematical description of the virtual space, its modification and generation of required incidents during the simulation drive. As for the projection of the animation, the most appropriate is the spatial system that covers the whole or most of the image seen by the motorman. It is important that multicolor systems are applied that form the energy characteristics of the image.

The movement of the simulator is one of the factors the make the whole virtual driving more real. Naturally, locomotives never accelerate and brake abruptly and do not need to negotiate sharp bends. Hence the motion actuators of the simulator system do not have to generate intense G-forces, as is in the case of e.g. an F1 simulation. The system however must be sufficiently complex to simulate a collision or a malfunction that triggers vibration in the motorman's compartment. The simulator should also be capable of simulating the inertia of the train i.e. the specific way of the interaction of the railcars with the locomotive as these railcars also generate certain g-forces. What the authors have in mind is the phenomenon of the surging of the railcars while braking and delaying while driving off. The system should send appropriate stimuli to the motorman's body through the motion of the simulator (Fig. 1). It is of paramount importance that the stimuli are synchronized with the projected images.

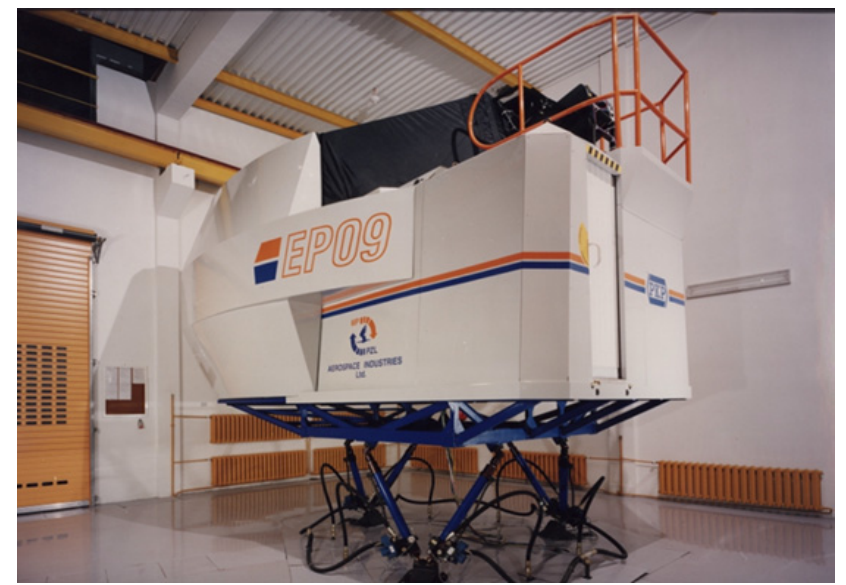

Figure 1: $\quad$ EP09 portable locomotive simulator [1]. 
A professional simulator, apart from stimulating the motorman's sense of sight should also generate the sense of motion (influence the motorman's labyrinth). The motion of the simulator cab where the motorman sits realizes this feature.

The virtual surroundings of a vehicle should be displayed with high resolution and possibly high frequency. Too low a resolution causes "pixelization" of an image and, consequently, a deterioration of the reality of the reflected objects. Additionally, a motorman is distracted when analyzing shapes or marks that are displayed in front of him. The minimum resolution of graphics should be $1024 \mathrm{x}$ 760 pixels, whereas the color palette should operate in the true color mode, i.e. with over 16 million colors. Images should be displayed with the frequency of 50 Hz. This will allow getting a feeling of smooth driving and will decrease the risk of a simulator disease. The high frequency is required due to the nature of the locomotive operation i.e. its high speeds. Additionally, in considering the vehicle inertia, one should ensure a distance of minimum $500 \mathrm{~m}$ between objects appearing in the virtual space.

Image presentation solutions generated by computers vary widely. The following systems may be distinguished $[2,3]$ :

- flat screen system;

- cylindrical screen system;

- spheroid screen system;

- systems of screens fitted in vehicle windows;

- a system with a screen fitted on a trainee's head - the solution resembles goggles or a helmet;

- a system based on a hemispherical screen placed close to the trainee's eyes.

The best solutions allow observing the simulated surroundings in all directions as desired by the motorman. This group includes cylindrical and spheroid systems. However, the structure requires large rooms and sophisticated display systems, as the graphics are projected onto a curved surface and it is necessary to use appropriate lenses. A cost effective and very efficient solution involves the use of systems with screens fitted in vehicle windows. This makes it possible to eliminate projectors and has a positive influence on the decrease of the size of the station. However, this solution requires some modifications in the simulator programs, which means that the vehicle motion should be separated from what is seen from the window. If shocks are simulated, the cabin should move and the image outside the window should move neutrally, which means that it should not be subject to the same shocks as the cab.

Basic requirements for a computer-aided locomotive driving simulator include:

- high detail level and sharpness of image - accurate reflection of the railroad infrastructure, including, signs, lights, obstacles etc. Ensuring reliable surroundings in a reasonably accurate manner;

- creation of large images - a high angle of view ensures obtaining realistic conditions and makes it possible for the motorman to control a vehicle in the most convenient manner; 
- $\quad$ simulation of atmospheric conditions - creation of most realistic atmospheric conditions as regards limited visibility, traction deterioration etc.;

- simple and quick change of scenarios -an easy change of the route, atmospheric conditions and, in particular, vehicle parameters such as weight load, power output;

- high quality and smoothness of the changing image - the graphics should be characterized by high resolution and appropriate refreshing frequency;

- simple operation and maintenance - operators should have their stations prepared in such a manner as to realize the adopted program of exercises without any additional training.

Additionally, a simulator should be characterized by reliability and resistance to damage caused by inappropriate operation by the trainees.

A simulator is a sophisticated technical device that has to be placed in a separate room. Its weight and measurements should be minimized, however, this should be done in such a manner as not to affect the quality of the motorman's work. Great weights also have a bad influence upon the power consumption by the systems that actuate the simulator. The best solution will be to use materials that are characterized by high strength and low weight. The very cabin should most accurately reflect a real cabin (fig. 2). The simulator structure should be characterized by optimum design and usability. The usability relates to solutions that additionally make it possible to change the design parameters of a locomotive or allow a total change of the vehicle type. This would be realized by reprogramming of controllers and/or quick introduction of some mechanical changes. This would have a favorable influence upon the quality of the training and testing processes, as it would give an opportunity to operate not only one type of a locomotive, but a certain group of locomotives.

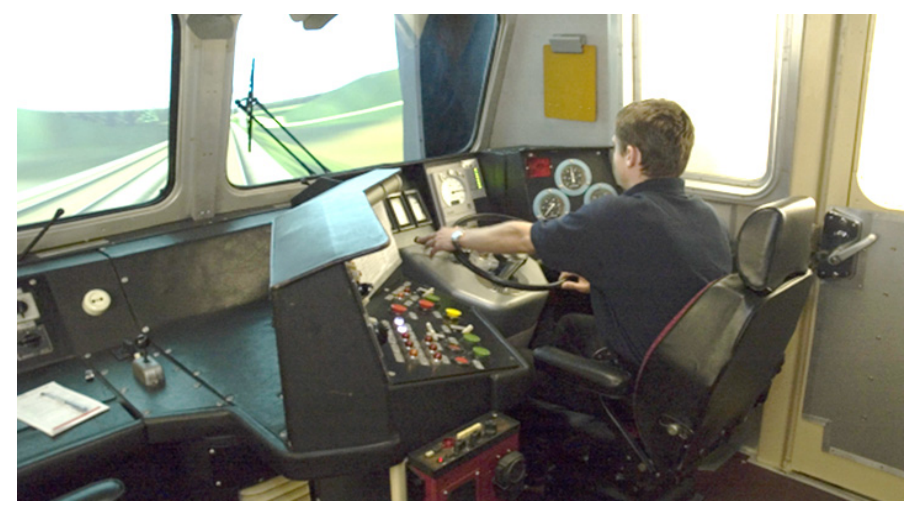

Figure 2: $\quad$ Interior of a simulator (simulates the EP09 locomotive) [4].

It is very important that a locomotive simulator reflects the most recent or modernized vehicles. Presently, various standards are introduced in EU member states and other countries of the world. They force using solutions that decrease energy consumption and have an impact upon the unification of the operation 
and locomotive equipment. For instance, directive No. 2008/57/EC of the European Parliament and Council as of 17 June 2008 relating to interoperability of the railroad system provides that commercial railroad in the entire territory of the European Union have to be fully compatible as regards vehicles, infrastructure and effective systems of exchange of information between various infrastructure administrators and railroad enterprises should be introduced [5]. To this end, Technical Specifications of Interoperability (TSI) are used. Thus, it is significant that a simulator be prepared for requirements for locomotives in a given country and its nearest vicinity.

\section{Image presentation}

In order to generate virtual reality in a simulator it is necessary to apply animation. Generally speaking, animation is a four-dimensional space. 3D graphics is based upon presentation of spatial elements on a plane. It uses description of translation, rotation around three axes perpendicular to one another and perspective view, analysis of visibility of a part of the object in consideration of the change of colors, hues, scale etc. Time is treated as the fourth dimension. The basis for the realization is the use of mathematical models. The more degrees of freedom that are used, the most favorable it is for quality of the simulator operation. Models should be characterized by the utmost quality compliance with properties of a simulated vehicle. The use of animation allows generating images in such a manner as to reflect vehicle motion and image changing on the screen (fig. 3).
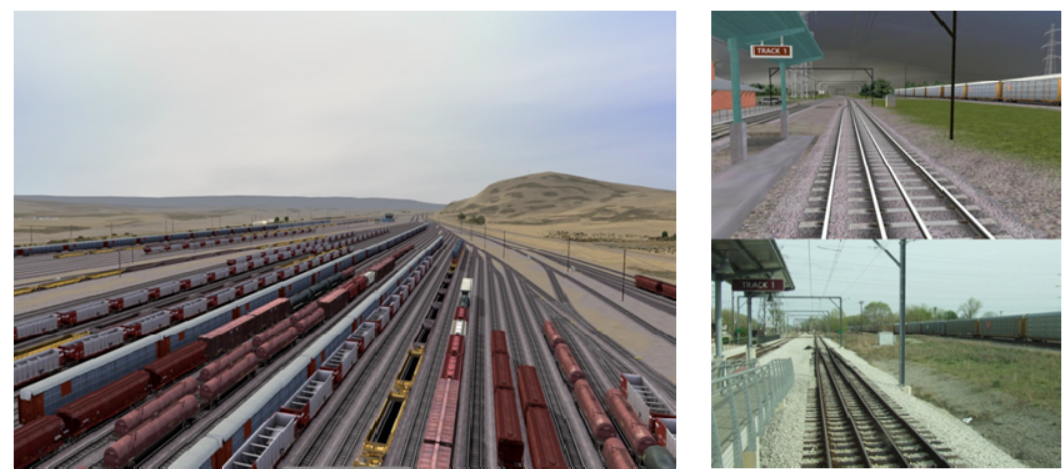

Figure 3: An example view of the virtual surroundings [6].

A locomotive driving visualization system makes it possible for the motorman to get oriented in the virtual space. The motorman's decisions (changes of the settings) constitute input data for the vehicle motion model. Upon calculation of the motion parameters, it is possible to determine the location and the speed in the virtual world. Apart from generating the most realistic possible image, mathematical models should create conditions triggering illusions for the human senses. This solution allows enhancing of some effects 
that have an impact upon making the artificial conditions realistic. The following factors are used for the enhancement of the spatiality illusion [2]:

- non-transparency of the image elements;

- use of perspective rules;

- mutual covering of the image elements;

- use of shadows, half-shadows and contrast changes.

When creating a simulation, each point must be described by three coordinates in the vehicle location system on the road and, preferably, with the space on which the operator's eyes are fixed. This way, the described surroundings are transformed into a flat image. Brightness of the displayed elements as well as the glowing and dispersion properties are important as well. One should also consider possible contamination in the room where the simulator is located. For some technical solutions the contamination may have adverse effects upon the operation of the system by creation of distortions of the displayed image. A human organism is more susceptible to dynamic effects than stroboscopic effects. It results from the fact that smooth motion in the virtual space is important without any image freeze phenomena and excessive distortions.

Creation and generation of an image in a simulator in an appropriate manner is connected with a phenomenon of linear perspective. It gives an opportunity to present $3 \mathrm{D}$ objects on a flat area and makes it possible to determine a mathematical form. The main features of the perspective that allow a 3D perception include: optic shortening (relative distances between objects seen from a certain distance) and depth (diminution of objects with a growth of distance). If a perspective is used in the image it is connected with use of an optical shortening. By shading, using contrasts, locating objects in space, overlapping of planes, comparison of colors and texture changes it is possible to obtain depth effects. Considering an observer's position in relation to an object, we may distinguish the following perspectives [2]:

- parallel;

- oblique;

- bird's eye view;

- curve-lined;

- atmospheric.

\section{Software and training}

A simulator should have as much information implemented (related to the largest number of parameters) as possible. The locomotive simulator database should include data relating to traction/motion properties of a vehicle, vehicle load, surroundings scenery, geometry, condition of the tracks and atmospheric conditions. It is important that a person supervising the training on a simulator have access to what a motorman sees, the decisions that he makes and how the decisions affect further operation (fig. 4). He should also be able to influence the ambient conditions, situation on the tracks and the simulation of damage and obstacles. 


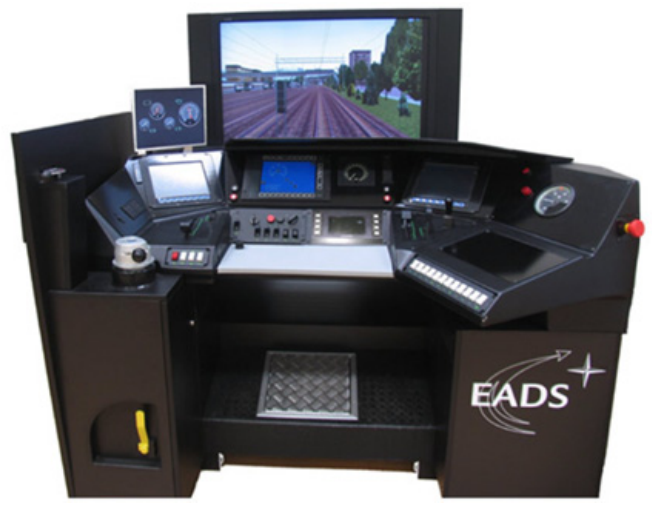

Figure 4: A station of the training supervisor (trainer) [7].

Virtual surroundings are of an extremely significant meaning for the motorman who performs the exercise. Elaboration of the exercise and care for details allow making the train drive more realistic and ensure a certain level of comfort. Near the tracks objects such as trees, houses etc. should be densely located. Their presence impacts on the feeling of speed and driving is not monotonous. If there are not objects around the track, a trainee may not feel the train speed at all. Sounds are also important in the simulator training.

A locomotive drive simulator not only provides an opportunity to learn how to drive and operate a locomotive but it should also train the motorman to react in aces of a direct hazard. The trainer is required to simulate a collision, track or train failure or other event, which forces a motorman to take some actions (fig. 5). In real operating conditions during the training, the above-mentioned situations rarely occur. The simulator operation gives an opportunity to prepare and learn appropriate reflexes in case of unexpected events. During a simulation

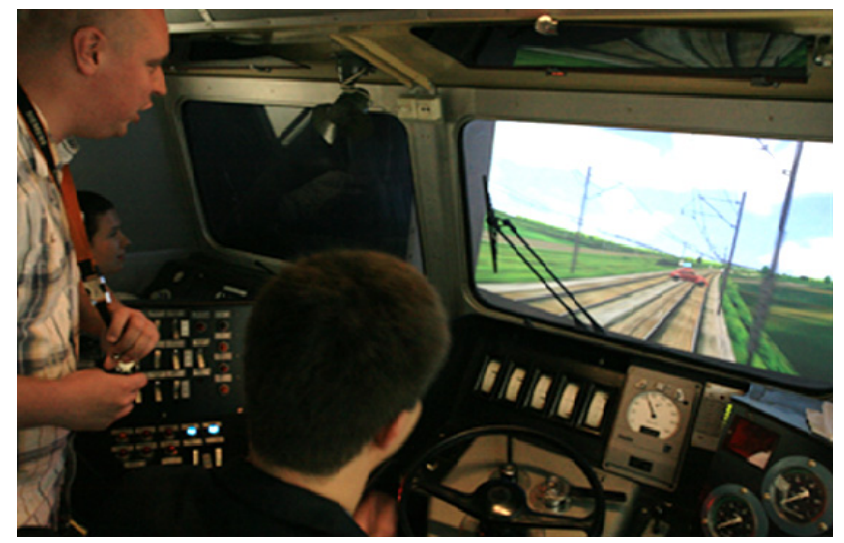

Figure 5: $\quad$ An example of a simulated obstacle during training [9]. 
of emergency situations, sound and cabin motion systems are extremely significant. For graphics generating computers, this task is not much of a problem. However, in order to obtain the highest possible degree of reality, the image should be accompanied by a sound simulating true sounds as well as a motion system that should reflect conditions in the motorman's cabin most accurately.

It is recommended that the simulator software and equipment provide an opportunity to collect and record as much data as possible. This is necessary not only for the control and assessment of the behavior of the trainee but also for the assessment of skills relating to economical driving. This is important from the point of view of additional training for motormen and teaching reflexes to young motormen. The Programs should be organized in such a way as to be able to show errors or inappropriate driving techniques leading to excessive consumption of energy and wear of the operating elements of the vehicle. A simulator should also be extendable. The extension involves modifications of the control panel [8]. Presently, locomotives use new solutions that improve driving safety and location etc. These are mostly adaptable devices, which means that they may be fitted in a vehicle without substantial mechanical modifications. An extended recording system and a possibility to model new communications solutions give an opportunity to make various analyses relating to railroad routes. An example could include the measurement of energy absorption for the set conditions of operation depending on the angle of the track slope in the longitudinal axis.

Moreover, for didactic purposes, the possibility of modification of the designed routes and even total rebuilding thereof is also significant. This will allow avoiding repetitive behavior and habits as the trainee will face new problems or problems structured in a different way.

\section{Negative effects of a driving simulator on the human organism}

Persons using simulators are sometimes prone to the simulator disease. Because of high speeds during the locomotive drive this inconvenience may occur more often than in the case of other types of simulators. This may be dangerous for the simulator users because, apart from the physical symptoms, psychical prejudice may take place. The disease is similar to a travel sickness. However, there are more causes for the disease. Apart from standard causes, the following may also be distinguished in the case of simulators $[2,10]$ :

- various issues with the balance organ, sense receptors and visual impressions of a motorman may appear if the motorman is accustomed to real driving conditions, in which he gained some experience;

- poor synchronization between the motor and visualization systems - there are some delays between the displayed image and the reaction of the mechanical systems that simulate the vehicle motion; 
- limited cab motion - the mechanical motion of the cab does not reflect the phenomena displayed and the cab generates loads and shocks with lesser intensity:

- interference in the image display - inappropriately matched colors, low frequency of graphics display, bad display angle, too rich a scenery or use of too strong illusions.

In order to eliminate causes of the discussed disease, good quality equipment should be used with the previously mentioned properties. Additionally, the problem should be considered at the model design stage (surroundings and colors).

\section{Conclusions}

Vehicle simulators allow transferring real conditions into the virtual world. Their application gives a lot of opportunities, however, it is not possible to ideally reflect all the factors appearing in the real world in an unambiguous manner. Rail vehicle simulators are characterized by specific requirements that differ from the recently very popular road vehicle simulators. To sum up, the most important requirements for a locomotive simulator may be formulated as follows:

- the simulator has to reflect a modern or a modernized locomotive based on modern solutions required by legal standards;

- the simulator should ensure the possibility of changing the design parameters of a locomotive or allowing a total change of the reflected vehicle type;

- the application of a precise and accurate model of the locomotive (and the whole train) dynamics and creation of models of operation of the on-board systems;

- the cabin interior should be similar to the real locomotive motorman compartment;

- the graphics should be of the best quality and provide the largest possible view for the motorman;

- the sounds and motion of the simulator should ensure sensations that most accurately resemble reality;

- the weight, size and energy consumption of a simulator cab should be selected in an optimum manner;

- the software should simulate various environmental conditions (selection of a season of the year and time of day and atmospheric conditions), allow modifications and creations of new routes, simulations of accidents and failures, reading and recording of as much data as possible relating to the driving techniques, energy absorption etc.;

- the system should include an instructor's ergonomic interface enabling a full review of the trainee's behavior and decisions as well as influence the events on the virtual drive route. 


\section{References}

[1] http://www.ai.com.pl

[2] Lozia Z.: Symulatory jazdy samochodem. Warszawa, WKŁ 2008.

[3] http://www.landersimulation.com/eng/

[4] http://www.csid.pl

[5] Białoń A., Gradowski P., Gryglas M.: Problematyka kształcenia kadr z wykorzystaniem symulatora systemu ERTMS do energooszczędnego prowadzenia pociągu. Pojazdy Szynowe 3/2011.

[6] http://www.orcarail.com

[7] http://www.cassidian.com/en_US

[8] Marciniak Z.: Lokomotywy elektryczne i spalinowe zmodernizowane w Polsce w ostatnich latach. Technika Transportu Szynowego 4/2011.

[9] http://www.plk-sa.pl

[10] Groen Eric L.; Bos Jelte E.: Simulator Sickness Depends on Frequency of the Simulator Motion Mismatch: An Observation. Teleoperators \& Virtual Environments, December 2008, Vol. 17 Issue 6, p 584-593. 